\title{
Implementation of a hospital-at-home (HAH) unit for hematological patients during the COVID-19 pandemic: safety and feasibility
}

\author{
Ignacio Gómez-Centurión ${ }^{1,2}$ (1) - Gillen Oarbeascoa ${ }^{1,2} \cdot$ María Carmen García ${ }^{2,3} \cdot$ María Carmen López Fresneña ${ }^{2,3}$. \\ María Josefa Martínez Carreño ${ }^{2,3}$. Vicente Escudero Vilaplana ${ }^{2,4}$. Eva González-Haba ${ }^{2,4} \cdot$ Rebeca Bailén $^{1,2}$. \\ Nieves Dorado ${ }^{1,2}$. Luis Miguel Juárez ${ }^{1,2}$. Gabriela Rodríguez Macías ${ }^{1,2}$ • Patricia Font López ${ }^{1,2}$. Cristina Encinas ${ }^{1,2}$. \\ Mariana Bastos-Oreiro ${ }^{1,2} \cdot$ Javier Anguita ${ }^{1,2} \cdot$ María Sanjurjo $^{2,4} \cdot$ José Luis Díez-Martin ${ }^{1,2,5} \cdot$ Mi Kwon $^{1,2}$
}

Received: 11 June 2021 / Revised: 7 September 2021 / Accepted: 7 September 2021 / Published online: 22 September 2021

(c) Japanese Society of Hematology 2021

\begin{abstract}
Background "Hospital-at-home" (HAH) programs have been shown to optimize resource utilization, shorten hospitalization and prevent nosocomial infection.

Methods We retrospectively analysed data regarding implementation of an HAH unit for caring patients with hematological malignancies in our center, during the COVID-19 pandemic.

Results Between January and November 2020, 105 patients were treated in the HAH unit for a total of 204 episodes. Nine patients with multiple myeloma (MM) received autologous HSCT (auto-HSCT). Three patients with acute myeloid leukemia (AML) received consolidation therapy, 32 patients underwent clinical and analytical monitoring, 20 were transplant recipients early discharged (5 auto-HSCT and 15 allo-HSCT) and 2 had received CART cells therapy. Azacitidine, bortezomib and carfilzomib were administered at home to 54 patients with AML, myelodysplastic syndrome (MDS) or MM. A median of 17 (IQR 13-19) days of admission per patient and a total of 239 visits to the Hematology day-care hospital were avoided. Overall, 28 patients (14\% of all episodes) needed admission to the hospital, 4 of them due to COVID-19.

Conclusions Implementation of a Hematology HAH unit was feasible and safe, and provided thorough advanced care to a high-risk population. Advanced care-at-home strategies can be crucial during times of COVID-19 to minimize treatment interruptions and reduce the risk of cross-infections.
\end{abstract}

Keywords Hospital-at-home $\cdot$ Hematology $\cdot$ COVID-19 $\cdot$ QOL

Ignacio Gómez-Centurión

igomezcenturion@gmail.com

1 Department of Hematology, Hospital General Universitario Gregorio Marañón, Doctor Esquerdo 46, 28007 Madrid, Spain

2 Gregorio Marañón Institute of Health Research, Madrid, Spain

3 Nursing Department, Hospital General Universitario Gregorio Marañón, Madrid, Spain

4 Department of Pharmacy, Hospital General Universitario Gregorio Marañón, Madrid, Spain

5 Universidad Complutense de Madrid, Madrid, Spain

\section{Introduction}

On December 2019, several cases of pneumonia with unknown etiology occurred in Wuhan, Hubei, China [1-3]. The pathogen was later identified as a novel coronavirus, named SARS-CoV-2 by the World Health Organization (WHO). The disease spread rapidly throughout China and then all over the world. On March 11, 2020, the WHO declared the coronavirus-19 disease (COVID-19), a global pandemic [4].

COVID-19 fatality can be as high as $15 \%$ in elderly population and patients with comorbidities [5]. Among them, patients with cancer are more susceptible to infection and have inferior outcomes than individuals without cancer [6-8]. Furthermore, patients with hematological malignancies and hematopoietic stem cell transplant (HSCT) recipients, are a potential high-risk population due to the immune 
impairment from their underlying disease, the use of immunosuppressive treatments, and the need of frequent hospital visits for clinical monitoring, treatment delivery and admission. Several studies have described high mortality rates of nearly $30 \%$ in this specific population [9-18].

The city of Madrid in Spain, has been at the epicenter of the COVID-19 pandemic with approximately 370,000 diagnosed cases and more than 19,000 deaths (with confirmed or suspected COVID-19) as of early December 2020 [19]. The rapid development of the outbreak resulted in an enormous pressure to the medical institutions and the healthcare system was dramatically overloaded by the end of March 2020. These circumstances caused cross-infections among patients and medical workers and also limited the care of chronically ill individuals and patients with cancer, including hematologic patients. Therefore, novel strategies were needed to allow an appropriate care and treatment delivery for these patients, and at the same time reduce the risk of exposure avoiding hospital admissions and day hospital visits, considering that medical centers became one of the main sources of infection.

Advanced care at home or "hospital-at-home" (HAH) programs have significantly spread out in the last years. These strategies have demonstrated to improve patients satisfaction, avoid unnecessary hospital admissions and reduce healthcare costs [20-23]. Furthermore, hematologic patients have been successfully treated with outpatient approaches worldwide, including Spanish centers [24]. However, data regarding the use of these programs during COVID-19 are scarce.

The objective of this study is to describe the implementation of an HAH unit for clinical and analytical monitoring, treatment delivery and specific at-home treatment programs, for patients with hematological malignancies and HSCT recipients, during the COVID-19 pandemic in our area.

\section{Patients and methods}

We retrospectively analysed the implementation of an $\mathrm{HAH}$ unit for caring patients with hematological malignancies in a tertiary hospital located in the city of Madrid.

In January 2020, an HAH unit was developed in our center as a multidisciplinary unit, including Hematology physicians and nurses, pharmacists, nutritionists, and social workers.

The main objectives of the HAH unit were as follows: to improve the perception of the therapeutic process and quality of life, to optimize resources, to decrease hospitalization length, and to potentially prevent nosocomial infections.

It was initially created for specific programs: at-home autologous HSCT for patients with multiple myeloma $(\mathrm{MM})$, at-home consolidation treatment for patients with acute leukemia and intravenous treatment delivery. Since March 2020, when COVID-19 cases rapidly increased in our center, the program expanded to other areas as part of our Hematology department strategy to ensure safe and high quality care for hematological patients during the outbreak.

Patients were included in the program if their residences were within a 30-min drive from our hospital and a caregiver was available at home $24 \mathrm{~h}$ a day. All patients gave informed consent before inclusion in the HAH unit.

Concerning the program flowchart, the hematologist prescribed the pharmacotherapeutic treatment in the hospital's electronic prescription program. Subsequently, the pharmacist checked the prescription. All hazardous medications or those whose stability conditions required it were prepared centrally in laminar flow cabinets in the pharmacy service.

Patients were visited in their homes by nurses for clinical monitoring, blood samples collection, line care and intravenous drugs administration, among other activities. The frequency of visits was determined by clinical criteria and treatment needs. Additionally, patients received regular phone calls from nurses and physicians and $24 \mathrm{~h}$ on-call medical coverage was ensured. An inpatient bed in the Hematology ward was always available for these patients in case they would need urgent evaluation to receive rapid care and avoid visits to the emergency room.

After the COVID-19 outbreak, prevention measures were adopted to avoid cross-infection between patients and nurses. Social distancing was recommended and patients and caregivers were advised to stay at home as much as possible. Patients were instructed to immediately communicate any suspected COVID-19 symptoms (fever, cough, or dyspnea), in which case visits were discontinued and patients were evaluated at the hospital. On the other hand, nurses were required to be asymptomatic the day of the visit, and nasopharyngeal swabs were collected during the peak of the outbreak to diagnose asymptomatic cases as well. Nurses wore FPP2 or FPP3 facemasks and hand hygiene was intensified.

\section{At-home autologous HSCT for patients with multiple myeloma}

Patients were admitted to the HSCT unit to receive the conditioning regimen with melphalan $\left(140\right.$ or $200 \mathrm{mg} / \mathrm{m}^{2}$, according to local protocols). Cryotherapy was used to prevent mucositis.

Antimicrobial prophylaxis was performed with oral levofloxacin $500 \mathrm{mg}$ qd, fluconazole $400 \mathrm{mg}$ qd and acyclovir $800 \mathrm{mg}$ bid from day 0 . Additionally, intravenous ceftriaxone $1 \mathrm{~g}$ qd was administered from day +4 until the first day of febrile neutropenia or until achieving an ANC of $1 \times 10^{9} / \mathrm{L}$. 
Engraftment syndrome primary prophylaxis with oral steroids was administered, according to previous reports $[25,26]$. Use of G-CSF was avoided.

Patients were discharged the day after stem cells were infused in case of clinical and analytical stability, were readmitted in case of severe refractory mucositis, persistent febrile neutropenia or due to physician decision and finally left the at-home program when stable engraftment was achieved and major complications were solved.

\section{At-home consolidation treatment for patients with acute leukemia}

Patients were admitted to the Hematology ward to receive high/intermediate dose cytarabine (HDAC/iDAC) and were discharged the day after the cycle was administered providing clinical and analytical stability.

Antimicrobial prophylaxis was performed with oral levofloxacin $500 \mathrm{mg}$ qd and acyclovir $800 \mathrm{mg}$ bid from day 0 . Additionally, intravenous ceftriaxone $1 \mathrm{~g}$ qd was administered from day +4 until the first day of febrile neutropenia or until achieving an ANC of $1 \times 10^{9} / \mathrm{L}$. Besides, antifungal prophylaxis with posaconazole was added in this high-risk population.

Patients were re-admitted in case of persistent febrile neutropenia or due to physician decision, and left the program when aplasia and major complications were solved.

\section{Other programs}

Clinical and analytical monitoring and electrolyte replacement were performed at home to patients with higher risk of infection, including patients recently treated with autologous or allogeneic HSCT and chimeric antigen receptor (CAR) $\mathrm{T}$ cells therapy.

Intravenous antimicrobial agents (including foscarnet) were administered at home either for infections treatment (including febrile neutropenia and bloodstream infections) or prophylaxis.

Venetoclax dose ramp-up was performed at home to patients with chronic lymphocytic leukemia (CLL) and acute myeloid leukemia (AML) with high risk for tumour lysis syndrome (TLS), with close analytical monitoring and intravenous hydration.

Azacitidine was administered at home to patients with AML and myelodysplastic syndrome (MDS).

Bortezomib and carfilzomib were administered at home to patients with MM.

\section{Definitions}

At-home episode was defined as any time a patient was included in the HAH unit for a specific program previously described until patient was discharged. One patient could have more than one episode, depending on the treatment delivered.

\section{Statistical analysis}

Quantitative variables were expressed as median and range. Qualitative variables were expressed as frequency and percentage. Analysis of normality was performed with the Kolmogorov-Smirnov test. Categorical data and proportions were analysed using chi-squared test or Fisher's exact test as required. Statistical analysis was performed using the IBM SPSS Statistic program version 25. Follow-up of patients was updated on December 2020.

\section{Results}

One hundred five patients were included in the HAH unit between January and November 2020, with a total of 204 at-home episodes (Table 1). Fifty (47\%) were female with a median age of 63 years (range 20-92).

Nine at-home autologous HSCT were performed in eight patients with MM (1 patient with high-risk MM received a tandem transplant). Six (75\%) were female with a median age of 60 years (range 43-66). All patients were discharged the day after infusion, except one with persistent vomiting, later discharged on day +5 . Median CD34(+) cells infused was $4.71 \times 10^{6} / \mathrm{kg}$ (range 4.32-11.7). Four patients developed grade 1 oral mucositis and 2 developed oral candidiasis. One patient needed in-hospital evaluation due to tachycardia, was diagnosed with atrial fibrillation, treated with oral drugs and discharged. Engraftment was achieved in a median time of 17 days (range 14-29) after transplant. Febrile neutropenia and engraftment syndrome rate was $0 \%$ and none of the patients were readmitted or diagnosed with SARS-CoV-2 infection. Median duration of the episodes was 18 days (range $13-20$ ).

Three patients were included in the at-home consolidation treatment program for AML: 2 to receive HDAC/iDAC cycle and 1 with high-risk acute promyelocytic leukemia (APL) to administer consolidation chemotherapy according to PETHEMA LPA-2017 protocol. They were discharged from the Hematology ward the day after the cycle was fully administered. All were readmitted: 1 with persistent febrile neutropenia in spite of intravenous antimicrobials and 2 due to SARS-CoV-2 related pneumonia. One patient developed COVID-19 symptoms $48 \mathrm{~h}$ after discharge from the Hematology ward and the second patient needed several evaluations in the hospital during admission in the HAH unit for transfusions. HAH unit nurses did not developed SARSCoV-2 symptoms simultaneously to these patients. Thus, SARS-CoV-2 cross-transmission between these two patients 
Table 1 Characteristics of patients and at-home episodes

\begin{tabular}{ll}
\hline Patients, $n$ & 105 \\
Episodes, $n$ & 204 \\
Median age, years (range) & $63(20-92)$ \\
Female, $n(\%)$ & $50(47 \%)$ \\
Diagnosis, $n(\%)$ & \\
MM & $35(33 \%)$ \\
AML & $27(26 \%)$ \\
NHL & $16(15 \%)$ \\
MDS & $15(14 \%)$ \\
ALL & $5(5 \%)$ \\
Others & $7(7 \%)$ \\
Program, episodes (\%) & \\
Admission avoidance programs & \\
At home autologous HSCT & $9(5 \%)$ \\
At home consolidation in acute leukemia & $3(1.5 \%)$ \\
Monitoring and electrolyte replacement (early dis- \\
charge) \\
Antimicrobial drugs delivery & $43(21 \%)$ \\
Venetoclax dose ramp-up & $16(8 \%)$ \\
Day-hospital avoidance programs & $4(2 \%)$ \\
Azacitidine & \\
Bortezomib & $83(40 \%)$ \\
Carfilzomib & $34(16.5 \%)$ \\
Re-admissions, $n$ (\%) & $12(6 \%)$ \\
SARS-CoV-2 related pneumonia & \\
Other causes & $4(2 \%)$ \\
Febrile neutropenia & $24(12 \%)$ \\
Progression of underlying disease & 7 \\
Other infections & 4 \\
Other & 8 \\
\hline & 5 \\
\hline
\end{tabular}

$A L L$ acute lymphoid leukemia, $A M L$ acute myeloid leukemia, $H S C T$ hematopoietic stem cell transplantation, $H L$ Hodgkin lymphoma, $M M$ multiple myeloma, $M D S$ Myelodysplastic syndrome, $N H L$ non-Hodgkin lymphoma

and HAH unit members was discarded. Median duration of an episode was 11 days (range 3-13).

Clinical and analytical monitoring or electrolyte replacement was performed at home in 32 patients (43 episodes). Five of these patients were autologous recipients who were early discharged (before achieving engraftment) from HSCT unit. Fifteen were allogeneic HSCT recipients and 2 had received CAR-T cells therapy. Eight patients were admitted to hospital during treatment delivery: 1 with CMV primoinfection, 2 with pneumonia, 2 with other clinical infections, 2 with symptomatic progression disease and 1 with SARS-CoV-2 related pneumonia. This later patient developed symptoms at the same time than her caregiver, and HAH unit nurses did not developed SARS-CoV-2 symptoms simultaneously to this patient. Therefore, cross-transmission with HAH unit members was reasonably discarded.
Fourteen patients (16 episodes) received antimicrobial agents at home for confirmed or suspected infections, 3 of them for bloodstream infections treatment. Five patients were admitted to hospital during treatment delivery: 2 with febrile neutropenia, 1 with symptomatic progression disease, 1 with CMV infection and 1 with post-traumatic central nervous system (CNS) hemorrhage. None of these patients were diagnosed with SARS-CoV-2 infection.

Four patients, 3 diagnosed with AML and 1 with CLL, with high risk for TLS, started treatment with venetoclax, and dose ramp-up was performed at home. Prophylactic hydration was administered and blood samples were collected to early diagnose TLS. Two patients developed analytical TLS resolved with hydration. None of these patients were admitted to hospital or diagnosed with SARS-CoV-2 infection.

Azacitidine was administered at home to 22 patients ( 83 episodes) with AML or MDS. Four patients needed admission during treatment delivery: 2 with febrile neutropenia, 1 with symptomatic disease progression and 1 with CNS hemorrhage. None of them were diagnosed with SARSCoV-2 infection.

Bortezomib was administered at home to 22 patients (34 episodes) with MM. One patient was admitted to hospital during treatment delivery due to acute limb ischemia and 1 patient was diagnosed with SARS-CoV-2 related pneumonia, at the same time than 2 other members of his family. HAH unit nurses did not develop SARS-CoV-2 symptoms simultaneously to these patients, and in consequence cross-transmission with HAH unit members was discarded.

Carfilzomib was administered at home to 8 patients (12 episodes) with MM. Only 1 scheduled treatment was not administered due to arterial hypertension, and no other incidences related to infusion were reported. One patient was admitted to hospital during treatment delivery due to fever and rash secondary to lenalidomide. None of the patients were diagnosed with SARS-CoV-2 infection.

Overall, more than 1000 visits were made to patient's homes by nurses from the HAH unit; as a consequence, a median of 17 (IQR 13-19) days of admission per patient to the Hematology ward or HSCT unit and a total 239 visits to the Hematology day-care hospital were avoided. This translated to a significant decrease of bed occupancy in the Hematology ward and HSCT unit during the peak of the pandemic (Figs. 1, 2).

In summary, 28 patients (14\% of all episodes) needed admission to the hospital while being treated by the $\mathrm{HAH}$ unit, 4 of them because of SARS-CoV-2 related pneumonia (Table 2). None of the personnel from the HAH unit developed SARS-CoV-2 infection while being actively caring patients at home. 


\section{Admissions in Hematology Ward during 2020}

100

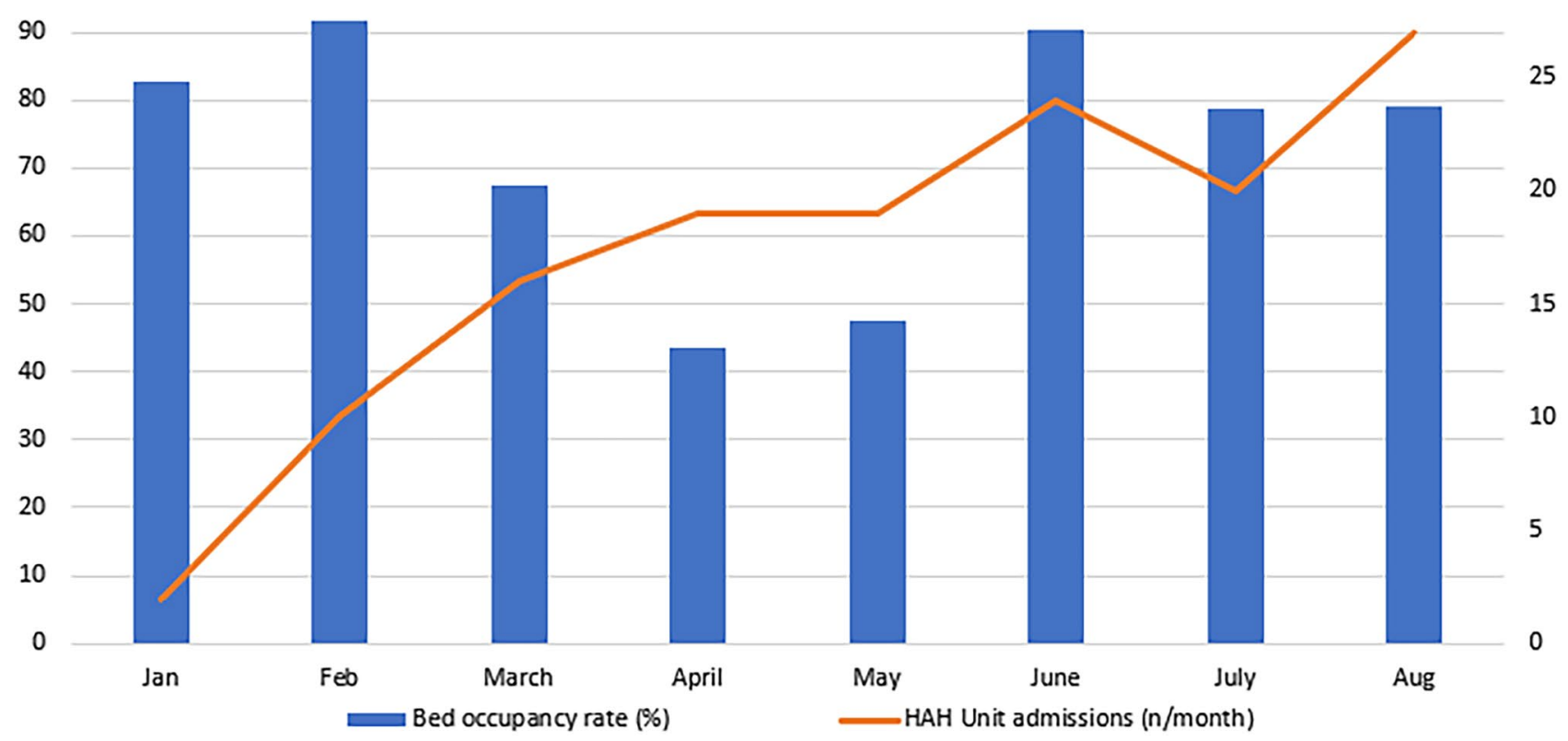

Fig. 1 Admissions in hematology ward during 2020

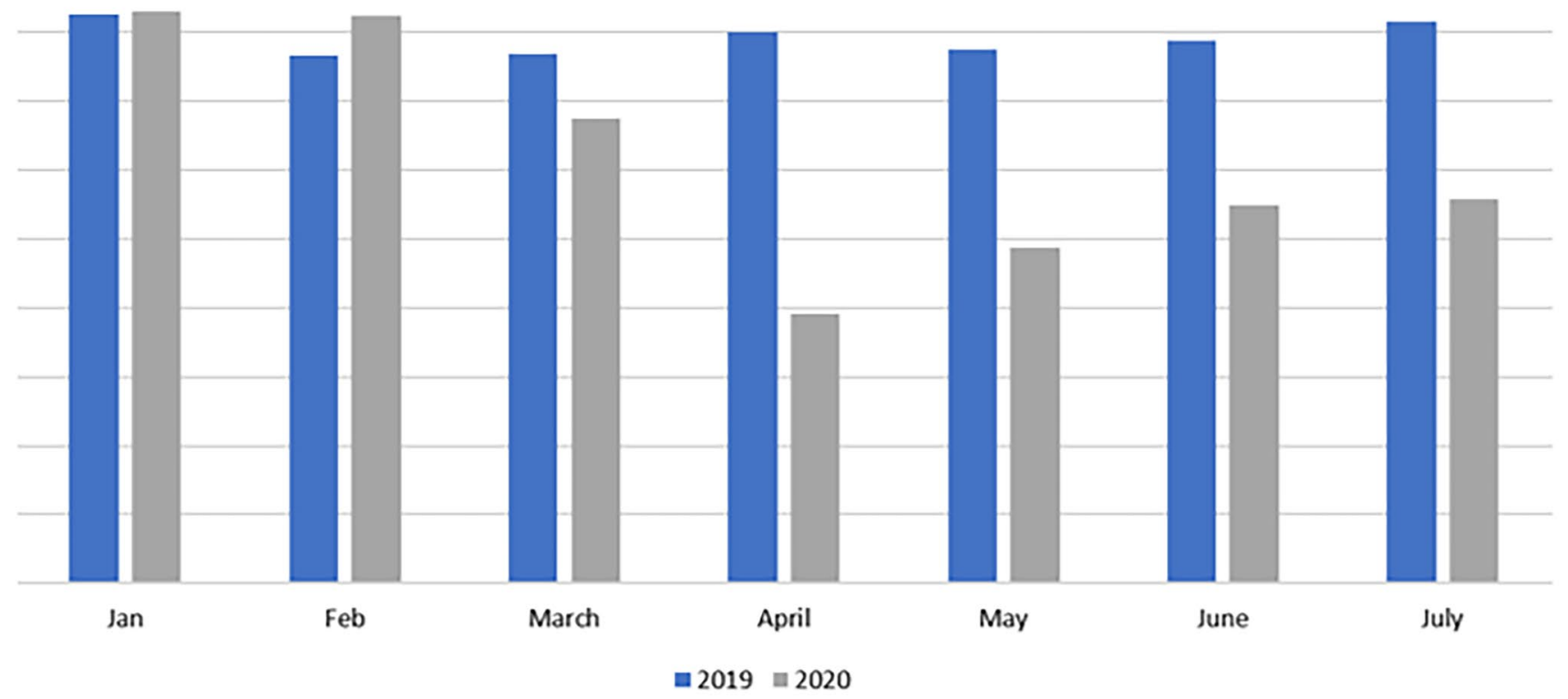

Fig. 2 Comparison between the admissions in hematology ward in 2019 and 2020

\section{Discussion}

In this study, we introduce our experience in the implementation of a Hematology $\mathrm{HAH}$ unit during the
COVID-19 pandemic for caring patients with hematological malignancies and HSCT recipients.

Since March 2020 when the WHO declared the coronavirus-19 disease (COVID-19) a global pandemic, Spain, and particularly the city of Madrid, has been at the 
Table 2 Characteristics and outcome of patients diagnosed with SARS-CoV-2 infection during admission in the HAH unit

\begin{tabular}{|c|c|c|c|c|}
\hline Patient & 1 & 2 & 3 & 4 \\
\hline Sex & Female & Male & Female & Female \\
\hline Age (years) & 47 & 73 & 40 & 79 \\
\hline Diagnosis & AML & AML & Hodgkin lymphoma & MM \\
\hline HAH program & $\begin{array}{l}\text { AML consolidation treat- } \\
\text { ment }\end{array}$ & $\begin{array}{l}\text { AML consolidation treat- } \\
\text { ment }\end{array}$ & $\begin{array}{l}\text { Clinical monitoring after } \\
\text { allo-HSCT }\end{array}$ & At-home Bortezomib \\
\hline $\begin{array}{l}\text { Admission in HAH unit } \\
\text { (date) }\end{array}$ & $26-03-2020$ & 24-03-2020 & $20-03-2020$ & 07-09-2020 \\
\hline Days in HAH unit & 3 & 10 & 18 & 18 \\
\hline $\begin{array}{l}\text { Number of visits from } \\
\text { HAH unit }\end{array}$ & 1 & 6 & 6 & 4 \\
\hline COVID-19 diagnosis (date) & 29-03-2020 & 03-04-2020 & 07-04-2020 & $25-09-2020$ \\
\hline Symptoms and signs & $\begin{array}{l}\text { Fever, cough and rhinor- } \\
\text { rhoea }\end{array}$ & Fever and cough & Fever and cough & Fever, cough and dyspnea \\
\hline Pneumonia & Yes & Yes & Yes & Yes \\
\hline Treatment & $\begin{array}{l}\text { Lopinavir/ritonavir + HQ/ } \\
\text { GCs/tocilizumab }\end{array}$ & HQ/GCs/tocilizumab & $\begin{array}{l}\text { HQ/GCs/tocilizumab/ } \\
\text { Anakinra/HI Plasma }\end{array}$ & $\begin{array}{l}\text { GCs/Remdesivir/Tocili- } \\
\text { zumab }\end{array}$ \\
\hline ICU admission & Yes & No & Yes & No \\
\hline Resolution & Yes & Yes & No & Yes \\
\hline
\end{tabular}

allo-HSCT allogeneic hematopoietic stem cell transplantation, AML acute myeloid leukemia, COVID-19 coronavirus-19 disease, GCs glucocorticoids, $H A H$ hospital at home, $H I$ hyperimmune, $H Q$ hydroxychloroquine, $I C U$ intensive care unit, $M M$ multiple myeloma

epicenter of the outbreak [15, 27]. During the months of March and April, healthcare system was dramatically overloaded, and hospitals became high risk places for crosstransmission. Social distancing was recommended to help reduce the infection rates. In this adverse context, maintaining appropriate patients care and particularly in cancer patients, became a challenge $[28,29]$. Consequently, new strategies to reduce admissions and hospital visits, especially in tertiary centers, and without jeopardizing patients care and safety, needed to be implemented.

Professional care at-home services have significantly increased worldwide lately. HAH programs improve patients quality of life and reduce healthcare costs [22, 23, 30]. Moreover, outpatient approaches avoid unnecessary admissions and day hospital visits. Additionally, previous studies have shown that outpatient strategies can be safe and effective in hematological patients, including HSCT recipients [24, 31-34]. Besides, given a choice between care in institution or at home, most people would prefer to stay at their own home [35]. In this direction, HAH services appear to be an interesting option during COVID-19 pandemic to keep social distancing and reduce hospital visits, particularly for high-risk population [36], and especially during high peaks.

In our centre, the development of a Hematology HAH unit concurred with COVID-19 pandemic emergency. Patients with diverse hematological conditions and varied treatments, were safely and effectively cared in their houses. More than 1000 visits were made to patient's homes by nurses, allowing high risk patients to continue with their treatments and reducing the exposure to healthcare establishments.

In adult population, the risk for severe COVID-19 increases with age, with older adults at highest risk specially if other comorbidities are associated [5,37]. Besides, patients with hematological malignancies and HSCT recipients are thought to be at higher risk due to underlying disease, use of immunosuppressive treatments and the need for regular visits to hospitals for different reasons, including treatment delivery and admission, with many reports showing a worse outcome in this particular population and supporting measures to minimize the risk of infection in these patients [9-15].

Without an available vaccine or consistent effective treatments, shielding has been extensively recommended, mainly to high-risk population. Besides, as previously exposed, healthcare system overload has caused difficulty in patient's care. Thus, there has been concern regarding treatment delay in patients with cancer and its impact on underlying disease outcome [8]. The use of an HAH program can overcome this issue, allowing patients to keep social distancing and to continue or even start new treatments for malignancies.

Previous studies have described an association between COVID-19 cases and recent hospital visits or admission in general population and specifically in patients with cancer and hematological malignancies, including HSCT recipients $[11,13,38]$. Furthermore, the number of infected healthcare workers has been proportionally high during the pandemic, showing that healthcare centres are high-risk 
places for transmission [39-42]. In this sense, avoiding visits to the hospital might have an impact in reducing the probability of infection.

In all, only four patients were diagnosed with COVID19 while receiving any treatment from our HAH unit, in spite of being an elderly and immunocompromised population. Although concerns about the safety of delivering treatments at home during high incidence of SARS-CoV-2 transmission in the community can be posed, taking appropriate measures such as intensifying barrier measures and regular PCR collection to healthcare, can overcome the risk of cross-transmission. In this sense, HAH strategies seem to reduce exposure to SARS-CoV-2 and might decrease the risk of infection of high-risk patients. Of note, none of the cases acquired the infection from $\mathrm{HAH}$ personnel. Nevertheless, this is a retrospective analysis, and prospective studies should be conducted to test this hypothesis.

Although cost-effectiveness analysis was not performed in this study, healthcare cost must have been reduced in consequence to previous reports [22, 23, 30], since a median of 17 days of hospitalization per patient and a total of 239 visits to the Hematology day-care hospital were avoided.

On the other hand, implementing these programs requires a remarkable effort from different areas and precisely coordinated actions between physicians, nurses, pharmacists, caregivers and patients, among others. However, we believe this effort is well worth it considering the extremely positive feedback from staff, caregivers and specially from patients [43]. Furthermore, spared hospitalization can be used for more complex procedures and decrease waiting lists.

Overall, implementation of a hematology HAH unit was feasible, safe and provided a thorough advanced care to a high-risk population. Admissions and visits to day hospital were significantly reduced and SARS-CoV-2 infection rate was low and probably not related with cross-transmission between patients and nurses from the unit.

Hence, we believe the use of advanced care at-home strategies can be crucial during times of COVID-19 to minimize treatment interruptions and reduce the risk of cross-infections.

Acknowledgements We thank the staff and nurses of all the hematology and transplant units, and families and caregivers, for their care and contributions to making this work possible.

Author contributions Conception and design: IGC, MK; provision of study materials or patients: all authors; collection and assembly of data: IGC, MK; data analysis and interpretation: all authors; manuscript writing: IGC, MK and final approval of manuscript: all authors.

Funding This work was partially supported by the Department of Health, Autonomous Community of Madrid grant COV20/CM, Ministry of Science and Innovation Grant PID2019-107545RB-I00, Ministry of Economy and Competitiveness ISCIII-FIS Grant FIS PI20/00521 co-financed by ERDF (FEDER) Funds from the European Commission, "A way of making Europe".

Availability of data and materials The datasets generated during and/or analysed during the current study are available from the corresponding author on reasonable request.

Code availability Not applicable.

\section{Declarations}

Conflict of interests The authors declare that they have no competing interests.

Ethics approval and consent to participate The study was approved by the institutional review board and all patients gave written informed consent for us to collect their clinical information before transplantation or initiation of treatment for research purposes according to the local ethics policy and the Declaration of Helsinki.

Consent for publication All patients provided written informed consent for publication.

\section{References}

1. Ren L-L, Wang Y-M, Wu Z-Q, Xiang Z-C, Guo L, Xu T, et al. Identification of a novel coronavirus causing severe pneumonia in human: a descriptive study. Chin Med J (Engl). 2020;133(9):1015-24.

2. Zhu N, Zhang D, Wang W, Li X, Yang B, Song J, et al. A novel coronavirus from patients with pneumonia in China, 2019. N Engl J Med. 2020;382(8):727-33.

3. Zhou P, Yang X-L, Wang X-G, Hu B, Zhang L, Zhang W, et al. A pneumonia outbreak associated with a new coronavirus of probable bat origin. Nature. 2020;579(7798):270-3.

4. Timeline: WHO's COVID-19 response. https://www.who.int/ emergencies/diseases/novel-coronavirus-2019/interactive-timel ine. Accessed 1 Dec 2020.

5. Onder G, Rezza G, Brusaferro S. Case-fatality rate and characteristics of patients dying in relation to COVID-19 in Italy. JAMA. 2020. https://doi.org/10.1001/jama.2020.4683.

6. Dai M, Liu D, Liu M, Zhou F, Li G, Chen Z, et al. Patients with cancer appear more vulnerable to SARS-CoV-2: a multicenter study during the COVID-19 outbreak. Cancer Discov. 2020;10(6):783-91.

7. Liang W, Guan W, Chen R, Wang W, Li J, Xu K, et al. Cancer patients in SARS-CoV-2 infection: a nationwide analysis in China. Lancet Oncol. 2020;21(3):335-7.

8. Wang H, Zhang L. Risk of COVID-19 for patients with cancer. Lancet Oncol. 2020;21(4): e181.

9. Fox TA, Troy-Barnes E, Kirkwood AA, Chan WY, Day JW, Chavda SJ, et al. Clinical outcomes and risk factors for severe COVID-19 in patients with haematological disorders receiving chemo- or immunotherapy. Br J Haematol. 2020. https://doi.org/ 10.1111/bjh.17027.

10. Booth S, Willan J, Wong H, Khan D, Farnell R, Hunter A, et al. Regional outcomes of severe acute respiratory syndrome coronavirus 2 infection in hospitalised patients with haematological malignancy. Eur J Haematol. 2020. https://covid19.elsevierpu re.com/fi/publications/regional-outcomes-of-severe-acute-respi ratory-syndrome-coronaviru. Accessed 15 Nov 2020. 
11. He W, Chen L, Chen L, Yuan G, Fang Y, Chen W, et al. COVID-19 in persons with haematological cancers. Leukemia. 2020;24:1-9.

12. Passamonti F, Cattaneo C, Arcaini L, Bruna R, Cavo M, Merli $\mathrm{F}$, et al. Clinical characteristics and risk factors associated with COVID-19 severity in patients with haematological malignancies in Italy: a retrospective, multicentre, cohort study. Lancet Haematol. 2020;7(10): e737-45.

13. Kanellopoulos A, Ahmed MZ, Kishore B, Lovell R, Horgan C, Shankara $\mathrm{P}$, et al. COVID-19 in bone marrow transplant recipients: reflecting on a single centre experience. Br J Haematol. 2020. https://www.ncbi.nlm.nih.gov/pmc/articles/PMC7283684/. Accessed 1 June 2020.

14. Malard F, Genthon A, Brissot E, van de Wyngaert Z, Marjanovic Z, Ikhlef S, et al. COVID-19 outcomes in patients with hematologic disease. Bone Marrow Transplant. 2020;6:1-5.

15. García-Suárez J, de la Cruz J, Cedillo Á, Llamas P, Duarte R, Jiménez-Yuste $\mathrm{V}$, et al. Impact of hematologic malignancy and type of cancer therapy on COVID-19 severity and mortality: lessons from a large population-based registry study. J Hematol Oncol. 2020;13(1):133.

16. Piñana JL, Martino R, García-García I, Parody R, Morales MD, Benzo G, et al. Risk factors and outcome of COVID-19 in patients with hematological malignancies. Exp Hematol Oncol. 2020;9:21.

17. Regalado-Artamendi I, Jiménez-Ubieto A, Hernández-Rivas JÁ, Navarro B, Núñez L, Alaez C, et al. Risk Factors and mortality of COVID-19 in patients with lymphoma: a multicenter study. HemaSphere. 2021;5(3): e538.

18. Martínez-López J, Mateos M-V, Encinas C, Sureda A, HernándezRivas JÁ, Lopez de la Guía A, et al. Multiple myeloma and SARSCoV-2 infection: clinical characteristics and prognostic factors of inpatient mortality. Blood Cancer J. 2020;10(10):103.

19. Comunicados COVID-19. Normativa y notas de prensa. Comunidad de Madrid. 2020. https://www.comunidad.madrid/servicios/ salud/comunicados-covid-19-normativa-notas-prensa. Accessed 15 Nov 2020.

20. Thomé B, Dykes A-K, Hallberg IR. Home care with regard to definition, care recipients, content and outcome: systematic literature review. J Clin Nurs. 2003;12(6):860-72.

21. Vaartio-Rajalin H, Fagerström L. Professional care at home: patient-centredness, interprofessionality and effectivity? A scoping review. Health Soc Care Community. 2019;27(4):e270-88.

22. Shepperd S, Iliffe S, Doll HA, Clarke MJ, Kalra L, Wilson AD, et al. Admission avoidance hospital at home. Cochrane Database Syst Rev. 2016;9: CD007491.

23. Dowell S, Moss G, Odedra K. Rapid response: a multiprofessional approach to hospital at home. Br J Nurs Mark Allen Publ. 2018;27(1):24-30.

24. Font $\mathrm{C}$, Fernández-Avilés F, Calderon C, García-Fernández T, Arab N, Pineda E, et al. Home management of acute medical complications in cancer patients: a prospective pilot study. Support Care Cancer Off J Multinatl Assoc Support Care Cancer. 2016;24(5):2129-37.

25. Gutiérrez-García G, Rovira M, Magnano L, Rosiñol L, Bataller A, Suárez-Lledó M, et al. Innovative strategies minimize engraftment syndrome in multiple myeloma patients with novel induction therapy following autologous hematopoietic stem cell transplantation. Bone Marrow Transplant. 2018;53(12):1541-7.

26. Mossad S, Kalaycio M, Sobecks R, Pohlman B, Andresen S, Avery $\mathrm{R}$, et al. Steroids prevent engraftment syndrome after autologous hematopoietic stem cell transplantation without increasing the risk of infection. Bone Marrow Transplant. 2005;35(4):375-81.

27. Datos sobre coronavirus. Comunidad de Madrid. 2020. https:// www.comunidad.madrid/gobierno/actualidad/datos-coronavirus. Accessed 1 Dec 2020
28. Chen H, Wu X, Wang W, Wang Q. When cancer encounters COVID-19 in China: what have we suffered, experienced and learned. Jpn J Clin Oncol. 2020;50(6):712-7.

29. Curigliano G. How to guarantee the best of care to patients with cancer during the COVID-19 epidemic: the Italian experience. Oncologist. 2020;25(6):463-7.

30. Hopp FP, Trzcinski E, Roth R, Deremo D, Fonger E, Chiv S, et al. Cost analysis of a novel interdisciplinary model for advanced illness management. Am J Hosp Palliat Care. 2015;32(3):350-6.

31. Svahn B-M, Remberger M, Myrbäck K-E, Holmberg K, Eriksson $\mathrm{B}$, Hentschke $\mathrm{P}$, et al. Home care during the pancytopenic phase after allogeneic hematopoietic stem cell transplantation is advantageous compared with hospital care. Blood. 2002; 100(13):4317-24.

32. Shah N, Cornelison AM, Saliba R, Ahmed S, Nieto YL, Bashir $\mathrm{Q}$, et al. Inpatient vs outpatient autologous hematopoietic stem cell transplantation for multiple myeloma. Eur J Haematol. 2017;99(6):532-5.

33. Vaughn JE, Buckley SA, Walter RB. Outpatient care of patients with acute myeloid leukemia: benefits, barriers, and future considerations. Leuk Res. 2016;45:53-8.

34. Vaughn JE, Othus M, Powell MA, Gardner KM, Rizzuto DL, Hendrie PC, et al. Resource utilization and safety of outpatient management following intensive induction or salvage chemotherapy for acute myeloid leukemia or myelodysplastic syndrome: a nonrandomized clinical comparative analysis. JAMA Oncol. 2015;1(8):1120-7.

35. GESIS - Leibniz Institute for the Social Sciences. https://www. gesis.org/en/eurobarometer-data-service/survey-series/standardspecial-eb/study-overview/eurobarometer-673-za-4561-may-jun2007. Accessed 1 Dec 2020.

36. Sung AD, Nichols KR, Chao NJ. House calls for stem cell transplant patients during the COVID-19 pandemic. Blood. 2020;136(3):370-1.

37. Yang J, Zheng Y, Gou X, Pu K, Chen Z, Guo Q, et al. Prevalence of comorbidities and its effects in patients infected with SARSCoV-2: a systematic review and meta-analysis. Int J Infect Dis IJID Off Publ Int Soc Infect Dis. 2020;94:91-5.

38. Mehta V, Goel S, Kabarriti R, Cole D, Goldfinger M, AcunaVillaorduna A, et al. Case fatality rate of cancer patients with COVID-19 in a New York Hospital System. Cancer Discov. 2020;10(7):935-41.

39. Barranco R, Ventura F. Covid-19 and infection in health-care workers: an emerging problem. Med Leg J. 2020;88(2):65-6.

40. CDC COVID-19 Response Team. Characteristics of health care personnel with COVID-19-United States, February 12-April 9, 2020. MMWR Morb Mortal Wkly Rep. 2020;69(15):477-81.

41. Lombardi A, Consonni D, Carugno M, Bozzi G, Mangioni D, Muscatello A, et al. Characteristics of 1573 healthcare workers who underwent nasopharyngeal swab testing for SARS-CoV-2 in Milan, Lombardy, Italy. Clin Microbiol Infect Off Publ Eur Soc Clin Microbiol Infect Dis. 2020;2:10.

42. Pérez-García F, Pérez-Zapata A, Arcos N, De la Mata M, Ortiz M, Simón E, et al. Severe acute respiratory coronavirus virus 2 (SARS-CoV-2) infection among hospital workers in a severely affected institution in Madrid, Spain: a surveillance cross-sectional study. Infect Control Hosp Epidemiol. 2020;29:1-7.

43. 118 días en la burbuja de Almudena: 'El estado de alarma me viene genial, estoy menos aislada'. ELMUNDO. 2020. https:// www.elmundo.es/papel/historias/2020/05/13/5ebbdd00fdddfff 4a88b4570.html. Accessed 1 Nov 2020.

Publisher's Note Springer Nature remains neutral with regard to jurisdictional claims in published maps and institutional affiliations. 